\title{
COVID-19 Unveiling Brain Calcifications
}

\author{
Sara H. Sadok ${ }^{1}$. João R. M. de Oliveira ${ }^{1}$ (I)
}

Received: 20 July 2021 / Accepted: 2 August 2021 / Published online: 15 August 2021

(c) The Author(s), under exclusive licence to Springer Science+Business Media, LLC, part of Springer Nature 2021

\begin{abstract}
Neurological symptoms in COVID-19 patients have attracted the interest of the scientific community, yet their mechanisms remain unknown. In some circumstances, the presence of neurological manifestations may result in an incidental diagnosis after a detailed investigation. In the present letter, we discuss a case published by Demir et al., in which the diagnosis of COVID-19 enabled the diagnosis of a rare neurological disorder, characterized by bilateral brain calcifications, commonly known by the eponym Fahr's syndrome. In addition, we report a case of primary brain calcifications unveiled by a suspected coronavirus infection.
\end{abstract}

Keywords Basal ganglia calcifications · COVID-19 · Fahr's disease · Primary familial brain calcifications

The recent evidence regarding the neurological symptoms associated with COVID-19 has intrigued the scientific community. It was highlighted that the virus might trigger neurological and psychiatric illness (Feature 2020). However, its mechanism has not yet been elucidated. The most prevalent neurological complications are related to cerebrovascular disease and altered mental states (Varatharaj et al. 2020). It is important to note that the neurological symptoms may be severe, regardless of mild pulmonary manifestations (Paterson et al. 2020).

In a recent manuscript published in Neurological Sciences, Demir (2020) attributed the incidental findings of bilateral brain calcifications in neuroimaging of a patient during SARS-CoV-2 infection to a condition known as Fahr's syndrome. In this case, there was an important contribution, since neurological symptoms enabled the incidental diagnosis of a rare neurological disorder.

The presence of bilateral symmetrical brain calcifications, in the absence of toxic, infectious, metabolic or traumatic causes, is often attributed to Fahr's. Although, the eponym derives its name from Karl Theodor Fahr, he was not the first to identify the disorder in 1930, as it was reported 80 years earlier by Delacour (Ferreira and Oliveira

João R. M. de Oliveira

joao.ricardo@ufpe.br

1 Laboratório de Imunopatologia Keizo Asami, Federal University of Pernambuco, Recife, Pernambuco 50670-901, Brazil
2018). In addition, regarding the nomenclature, the term Fahr's syndrome is often associated with secondary causes, whereas Fahr's disease is related to primary etiology.

In our research group, we revised the clinical records of a 49-year-old male patient, unvaccinated, with a confirmed laboratory diagnosis of coronavirus disease (immunological diagnosis). In addition, chest computed tomography (CT) evaluation was performed (Fig. 1). The patient presented with seizures, with no known comorbidities, followed by cardiac arrest. The investigation proceeded

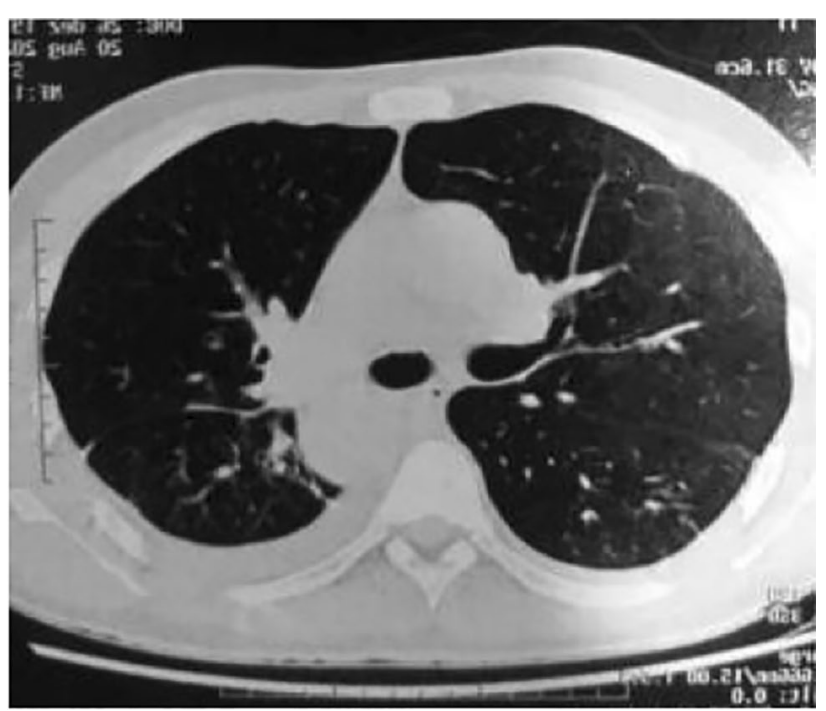

Fig. 1 Chest computed tomography (CT) 
Fig. 2 Brain CT showing bilateral brain calcifications

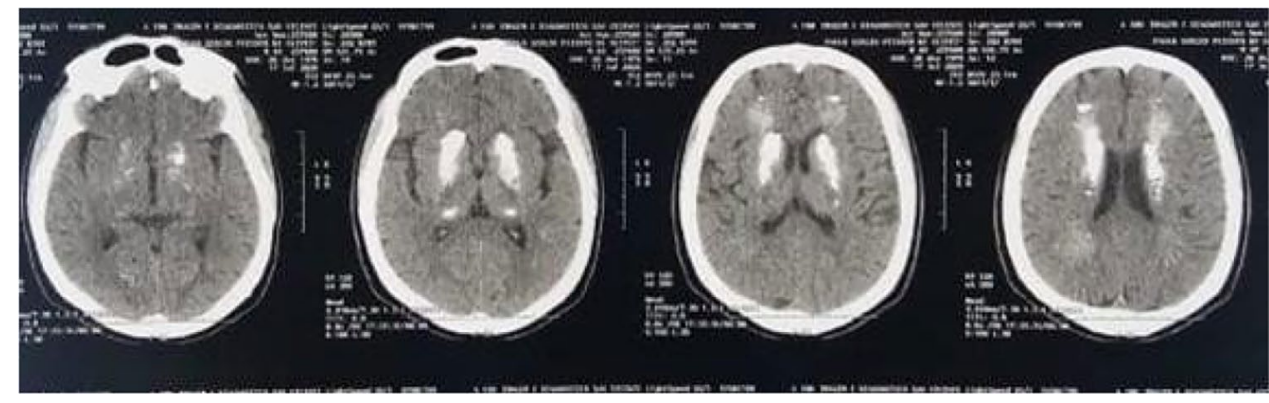

with neuroimaging that revealed bilateral brain calcifications (as shown in Fig. 2). Unlike the case described by Demir (2020), where ectopic calcinosis was linked to a hormonal etiology, our patient would be diagnosed as having primary or idiopathic brain calcifications. After discharge and follow-up, the patient's immunization status has changed. Concerning neurological manifestations, he is now asymptomatic.

As evidenced by this observation, the neurological manifestations are not always attributable to the infection or to its immune response. In addition, there are also situations in which a previously unknown disease is revealed or worsened by SARS-CoV-2 or where the hospitalization represents an opportunity for a proper workup for the patient.

The impact of neurological disease related to the coronavirus infection is not limited to acute illness and affects both the central and peripheral nervous system. We would like to highlight that along with brain disorder resulting from the illness, it is important to consider, for a differential diagnosis, prior unknown disease and concomitant nosocomial infections. The hospitalization and the extensive investigation therefore result in the diagnosis of these conditions.

Considering the challenges and the global burden of the COVID-19 pandemic, both researchers and physicians should continue efforts to flatten the infection curve, while actively searching for possible chronic consequences arising from the disorder.

Authorship Contributions Oliveira JRM (João Ricardo Mendes de Oliveira $\mathrm{MD}, \mathrm{PhD}$ ): conceptualization; critical revision of the manuscript. Sadok SH (Sara Hadj Sadok MD): Data curation; formal analysis; writing of the original draft. Both authors read and approved the final version to be published.

Funding The authors received no financial support.

\section{Declarations}

Ethics Approval and Consent to Participate Informed consent was obtained from the patient and the family.

Consent for Publication All authors have read and approved the final version of this manuscript.

The manuscript is original and has not been published previously and is not currently being considered for publication elsewhere.

Competing Interests The authors declare no competing interests or ethical issues.

\section{References}

Demir G (2020) Fahr's syndrome presenting with seizures in SARS-CoV-2 (COVID-19) pneumonia - a case report. 2(195):3063-3065

Feature N (2020) How COVID-19 can damage the brain, pp 2-7

Ferreira LD, de Oliveira JRM (2018) The need for consensus on primary familial brain calcification nomenclature. J Neuropsychiatry Clin Neurosci 30(4):291-293. https://doi.org/10.1176/appi. neuropsych. 18030044

Paterson RW et al (2020) The emerging spectrum of COVID-19 neurology: clinical, radiological and laboratory findings, pp 2-37

Varatharaj A et al (2020) Neurological and neuropsychiatric complications of COVID-19 in 153 patients : a UK-wide surveillance study. 7:875-882. https://doi.org/10.1016/S2215-0366(20)30287-X

Publisher's Note Springer Nature remains neutral with regard to jurisdictional claims in published maps and institutional affiliations. 